\title{
Nonlinear effects of carbon nanotube coated single mode optical fiber gratings
}

\section{Efectos no lineales en redes de fibra óptica cubiertas con nanotubos de carbono}

\author{
Guillermo E. Villanueva(1,*), Michael B. Jakubinek(2), Benoit Simard(2), Claudio J. Otón(1), \\ Li-Yang Shao(3), Pere Pérez-Millán(1), Jacques Albert(3)
}

1. Nanophotonics Technology Center, Universidad Politécnica de Valencia, Camino de Vera s/n, 46022 Valencia, Spain. 2. Steacie Institute for Molecular Sciences, National Research Council Canada, 100 Sussex Dr., Ottawa ON K1A 0R6, Canada.

3. Advanced Photonic Components Group, Carleton University, 1125 Colonel By Drive, Ottawa ON K1S 5B6, Canada.

(*)Email: guivilib@ntc.upv.es

Recibido / Received: 06/09/2011. Aceptado / Accepted: 10/01/2012.

\begin{abstract}
:
Single-wall carbon nanotube deposition on the cladding of optical fibers has been carried out to fabricate an all-fiber nonlinear device. Two different nanotube deposition techniques were studied. The first consisted of repeatedly immersing the optical fiber into a nanotube supension, increasing the thickness of the coating in each step. The second deposition involved wrapping a thin film of nanotubes around the optical fiber. For both cases, interaction of transmitted light through the fiber core with the external coating was assisted by the cladding mode resonances of a tilted fiber Bragg grating. Ultrafast nonlinear effects of the nanotube-coated fiber were measured by means of a pumpprobe pulses experiment.
\end{abstract}

Key words: Fiber Bragg Grating, Non-Linear Materials, Fiber Devices.

\section{RESUMEN:}

Se han depositado nanotubos de carbonos single-walled en las cubiertas de fibras ópticas para conformar un dispositivo óptico no lineal todo-fibra. Para la deposición se han estudiado dos técnicas. La primera consistió en la inmersión repetida de la fibra en una dispersión de nanotubos, incrementando el grosor de la capa de nanotubos con cada nueva inmersión. El segundo método se basó en el enrollado de una fina capa de nanotubos alrededor de la fibra óptica. Para ambos casos, la interacción de la luz transmitida con la capa de nanotubos fue asistida por medio de resonancias de modos de cladding de una red inclinada de Bragg. Gracias a un montaje pump-probe se pudo medir efectos no lineales ultrarrápidos en el dispositivo confeccionado.

Palabras clave: Redes de Fibra de Bragg, Materiales No Lineales, Dispositivos de Fibra.

\section{REFERENCIAS Y ENLACES / REFERENCES AND LINKS}

[1]. Y. Sakakibara, A. G. Rozhin, H. Kataura, Y. Achiba, M. Tokumoto, “Carbon nanotube-poly(vinylalcohol) nanocomposite film devices: applications for femtosecond fiber laser mode lockers and optical amplifier noise suppressors", Jpn. J. Appl. Phys. 44, 1621-1625 (2005).

[2]. K. K. Chow, S. Yamashita, Y. Song, "A widely tunable wavelength converter based on nonlinear polarization rotation in a carbon-nanotube-deposited D-shaped fiber", Opt. Express 17, 7664-7669 (2009).

[3]. S. Y. Set, H. Yaguchi, Y. Tanaka, M. Jablonski, "Ultrafast fiber pulsed lasers incorporating carbon nanotubes", IEEE J. Sel. Top. Quant. 10, 137-146 (2004). 
[4]. K. K. Chow, M. Tsuji, S. Yamashita, "Single-walled carbon-nanotube-deposited tapered fiber for fourwave mixing based wavelength conversion", Appl. Phys. Lett. 96, 061104 (2010).

[5]. K. K. Chow, S. Yamashita, "Four-wave mixing in a single-walled carbon-nanotube-deposited D-shaped fiber and its application in tunable wavelength conversion", Opt. Express 17, 15608-15613 (2009).

[6]. C. Chan, C. Chen, A. Jafari, A. Laronche, D. J. Thomson, J. Albert, "Optical fiber refractometer using narrowband cladding-mode resonance shifts", Appl. Opt. 46, 1142-1149 (2007).

[7]. C. T. Kingstone, Z. J. Jakubek, S. Dénommée, B. Simard, "Efficient laser synthesis of single-walled carbon nanotubes through laser heating of the condensing vaporization plume", Carbon 42, 16571664 (2004).

[8]. M. B. Jakubinek, M. B. Johnson, M. A. White, J. Guan, B. Simard, "Novel method to produce singlewalled carbon nanotube films and their thermal and electrical properties", J. Nanosci. Nanotechno. 10, 8151-8157 (2010).

[9]. T. Vallaitis, C. Koos, R. Bonk, W. Freude, M. Laemmlin, C. Meuer, D. Bimberg, J. Leuthold, "Slow and fast dynamics of gain and phase in a quantum dot semiconductor optical amplifier", Opt. Express 16, 170-178 (2008).

\section{Introduction}

Single-wall carbon nanotubes (SWNTs) have found innovative applications in the optical field due to their nonlinear properties in the near IR. Depending on the chirality of SWNTs, they can be either metallic or semiconducting, the latter being the desired behavior for nonlinear optical performance. Many optical applications could benefit from using SWNTs as nonlinear materials, such as noise suppression, wavelength conversion and passive mode-locking [1-3]. A challenge when designing SWNT-based devices is incorporating the nanotubes in such a way as to enhance their interaction with light. One common method involves dispersing SWNTs in a solvent and spraying onto substrates in order to produce a nanotube film in the middle of a light beam [3]. Some inherent drawbacks in these configurations are the need for alignment and focusing stages, low SWNT burn thresholds and low nonlinear interaction length. Solutions that overcome these challenges are based on SWNT deposition onto optical fibers. Tapered and Dshaped fibers have been proposed to take advantage of the evanescent field interaction with SWNTs, distributing that interaction along the fiber length $[4,5]$. Nevertheless, manufacturing and handling these kinds of fibers is quite complex and delicate. In this work we propose for the first time SWNT deposition on standard optical fiber cladding for nonlinear applications. Interaction of light propagating through the fiber core with the outer SWNT coating is achieved via cladding mode resonances in a tilted fiber Bragg grating (TFBG).
Two nanotube deposition methods have been carried out: dip-coating of the optical fiber in a SWNT suspension and wrapping of a SWNT film around the optical fiber.

\section{Operating principle}

A TFBG is a kind of grating in which the index modulation planes are not orthogonal to the fiber axis, but form a particular tilt angle. This inclination enhances the coupling of light from core mode to counter-propagating cladding mode resonances. Consequently, the grating transmission response is a multi-notch response consisting of numerous cladding mode resonances in addition to the core mode resonance (see Fig. 1(a)). The transverse mode profile of cladding modes spreads to the cladding of the fiber and interacts with the outer medium interface. Therefore cladding mode resonance frequencies depend on the refractive index of the outer medium in addition to other parameters. This feature can be exploited for refractive sensing applications [6]. In our device, the external coating is a layer of SWNTs, and interaction with light is allowed through cladding mode resonances. As SWNTs are a nonlinear material for optical wavelengths, the outer refractive index depends on the light intensity and an intensity-dependent TFBG transmission response is obtained. Fig. 1(b) depicts the SWNT-coated optical fiber structure. 
a)

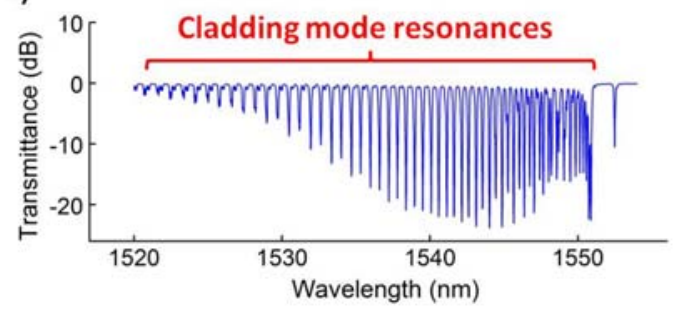

b)

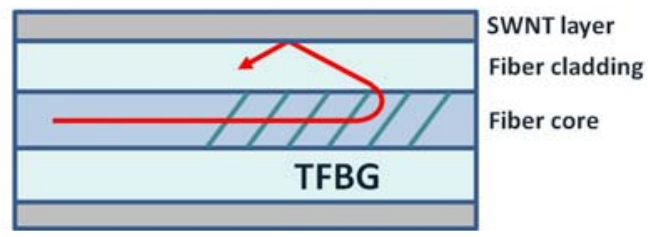

Fig. 1: (a) Measured linear transmission response of a 4 degree tilted fiber Bragg grating. (b) Nanotube-coated TFBG structure. Light for which the wavelength matches a cladding mode resonance couples to a cladding mode, interacting with the outer SWNT layer.

\section{SWNT deposition process}

Deposition of the SWNT coating was done either by dip-coating in a suspension of SWNTs in N,Ndimethylformamide (DMF) (dip-coated), or by collecting sections of a freely floating SWNT film around the optical fiber cladding (filmwrapped). Both approaches used high purity SWNTs $(\sim 1.3 \mathrm{~nm}$ in diameter and $>1 \mu \mathrm{m}$ in length) produced by a laser-oven method [7], and deposited onto single mode fibers with a TFBG written in them. Both gratings had a length of $20 \mathrm{~mm}$, a tilt angle of 4 degrees and a center wavelength of $1552 \mathrm{~nm}$. They were fabricated using a phase mask and a $248 \mathrm{~nm}$ pulsed irradiation from a $\mathrm{KrF}$ excimer laser. An example of TFBG transmittance in this study, measured when injecting light from a $1550 \mathrm{~nm}$ centered broadband source into an uncoated fiber, is shown in Fig. 1(a).

For dip-coating, $\sim 10 \mathrm{mg}$ SWNTs were first functionalized by bath sonication ( $2 \mathrm{hr}$ ) in $70 \%$ nitric acid. These SWNTs were recovered by filtration, rinsed with nanopure water $(>18 \mathrm{M} \Omega$ $\mathrm{cm}$ ) and by sonication ( $2 \mathrm{hr}$ ) in nanopure water, and then re-dispersed by sonication in $10 \mathrm{~mL}$ DMF. After the acid-treatment the SWNTs disperse readily in DMF producing a black suspension. The optical fibers were then coated by dipping the section containing the TFBG, sequentially, in a $0.1 \mathrm{wt} \%$ aqueous solution of 3aminopropyltriethoxysilane (APTES), followed by the SWNT/DMF suspension, and finally in pure water. APTES has been used in the preparation of CNT networks on glass, silicon, and PET. In that approach submerging the substrate in an ATPES solution results in formation of an amino-terminated, silanized surface. This surface is positively charged and the $\mathrm{COOH}$-functionalized SWNTs adsorb readily. The last step of water immersion was intended to homogenize the SWNT distribution and provide a well-known external medium when measuring TFBG transmission spectra. Repeated dipping cycles were used to increase the amount of SWNT coating and the deposition was monitored by measuring the transmission spectrum of the grating after each of ten dipping cycles. Cladding mode resonances are related to the outer medium properties, therefore the amount of deposited SWNTs will be reflected in these resonances. Figure 2 shows, in detail, one high order cladding mode centered at $1523.6 \mathrm{~nm}$ for each dipping step. To quantitatively compare resonance wavelength shifts and depth changes, all spectra have been normalized in amplitude and wavelength to the Bragg mode resonance (which is inherently immune to coating and external media changes). A clear shift $(\sim 67 \mathrm{pm})$ of the cladding mode resonance towards higher wavelength is observed, in addition to a decrease in resonance depth. Monitoring this relative shift of cladding mode resonance wavelength provides a direct indicator of SWNT coating thickness for consecutive dip-coating cycles. In Fig. 3 these wavelength shifts and modulation depth variations are plotted. On the one hand, a clear increasing monotonic trend is observed for the wavelength shift. On the other hand, concerning the transmittance shifts, the trend is not so clear, appearing a decreasing hop from the initial state to the first dipping step, and an erratic value for the eighth step.

For the film-wrapped TFBG, a floating SWNT film was first prepared as previously described [8]. In this case SWNTs ( $4 \mathrm{mg} / \mathrm{L}$ ) were dispersed in a $2 \mathrm{wt} \%$ aqueous sodium cholate solution, followed by production of a thin film by vacuum filtration of $\sim 10 \mathrm{~mL}$ through a cellulose acetate filter membrane ( $47 \mathrm{~mm}$ diameter, $0.22 \mu \mathrm{m}$ pore 


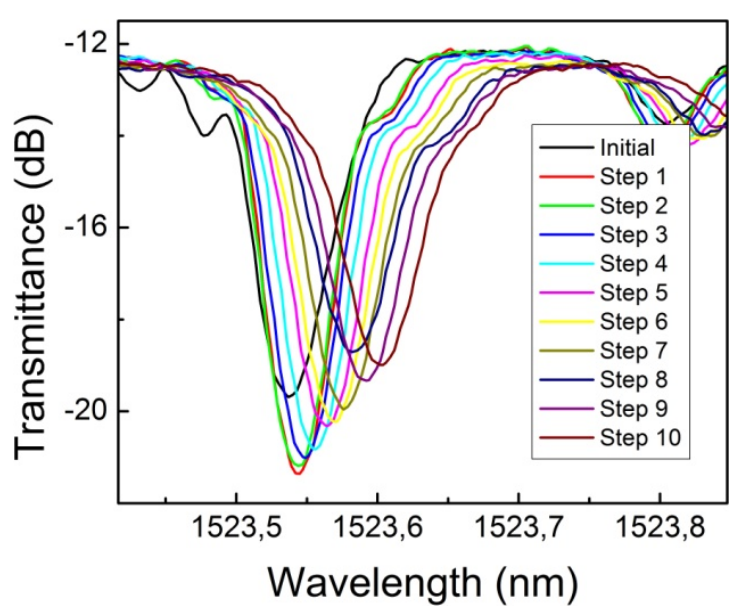

Fig. 2: Cladding mode resonance monitored for one to ten dip-coating cycles. All responses are wavelength and amplitude equalized with regard to the Bragg resonance.

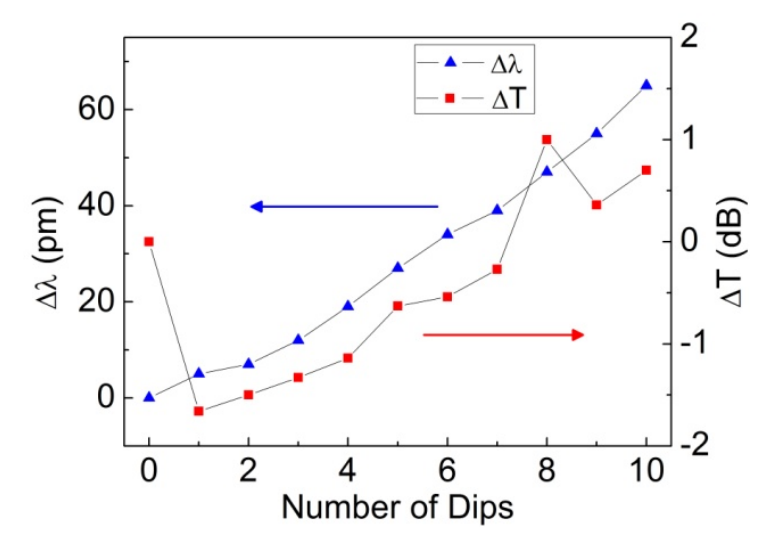

Fig. 3: Relative wavelength $(\Delta \lambda)$ and transmittance $(\Delta T)$ shifts of the cladding mode monitored versus the number of dips. These relative shifts are referred to the initial wavelength and transmittance values of the cladding mode resonance before any dipping step, which are $1523.537 \mathrm{~nm}$ and $-19.69 \mathrm{~dB}$ respectively.
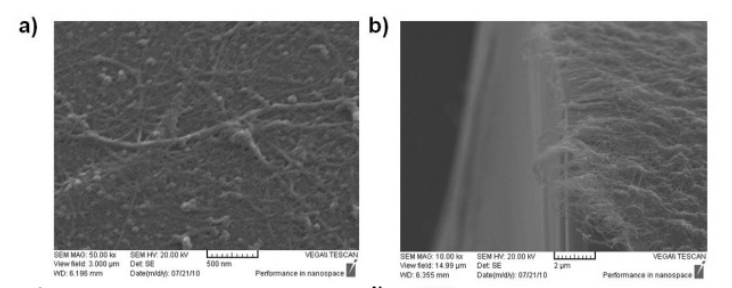

c)
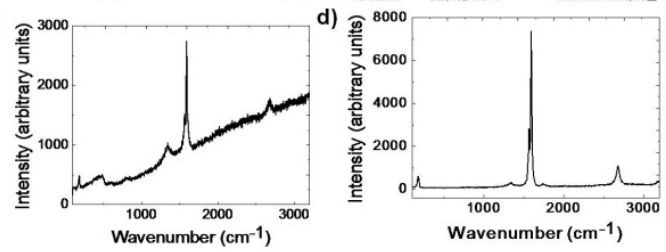

Fig. 4: SEM images of deposited nanotubes on the optical fiber cladding for (a) dip-coating and (b) wrapping techniques. Raman spectroscopy for (c) dip-coating and (d) wrapping techniques. size), and then detachment of the film by submersion in water. Part of the floating film was collected by slowly lifting and rotating the optical fiber as it was pulled through the film in order to obtain a SWNT coating over the TFBG. The film tears as it is lifted away from the water surface but the method still allows for efficient collection of a much thicker coating, which is visibly dark grey.

\section{Experimental results}

Both SWNT coatings were examined by SEM imaging and Raman spectroscopy, and the results are shown in Fig. 4, proving the presence of nanotubes on the fiber surface. From SEM images and variation of the intensity of the Raman spectra from location-to-location, we can deduce that dip-coating provides a thinner and more homogeneous layer of SWNTs on the fiber. In contrast the wrapping technique produces a much thicker coating, but with less control of the uniformity of the wrapping.

An experiment is performed to demonstrate the interaction of light with the SWNT coating via TFBG cladding mode resonances. Nonlinear effects were measured with a pump-probe phase-sensitive set-up described elsewhere [9]. Fig. 5 shows the scheme of the nonlinear set-up. An initial optical pulse is processed to obtain a three-pulse train; a pump, a probe and a reference pulse. The reference and the probe pulses are modulated by means of acousto-optic modulators with slightly different frequencies. This train is launched to the sample, in our work, the CNT-coated TFBG. After the sample, the optical train enters an interferometer in order to beat the reference and the probe pulses. The difference frequency component is detected through a lock-in amplifier.

In our experiment, the pulsed light injected into the TFBG had $-3.5 \mathrm{dBm}, 1535 \mathrm{~nm}$ and $1 \mathrm{ps}$ of average power, wavelength and pulse duration respectively, providing $22.5 \mathrm{~W}$ of peak power in the fiber. The 1 ps duration determines the measurement resolution. The optical fiber coated by the wrapping method was measured since it provides a thicker coating compared to dip-coating and, therefore, larger nonlinear 


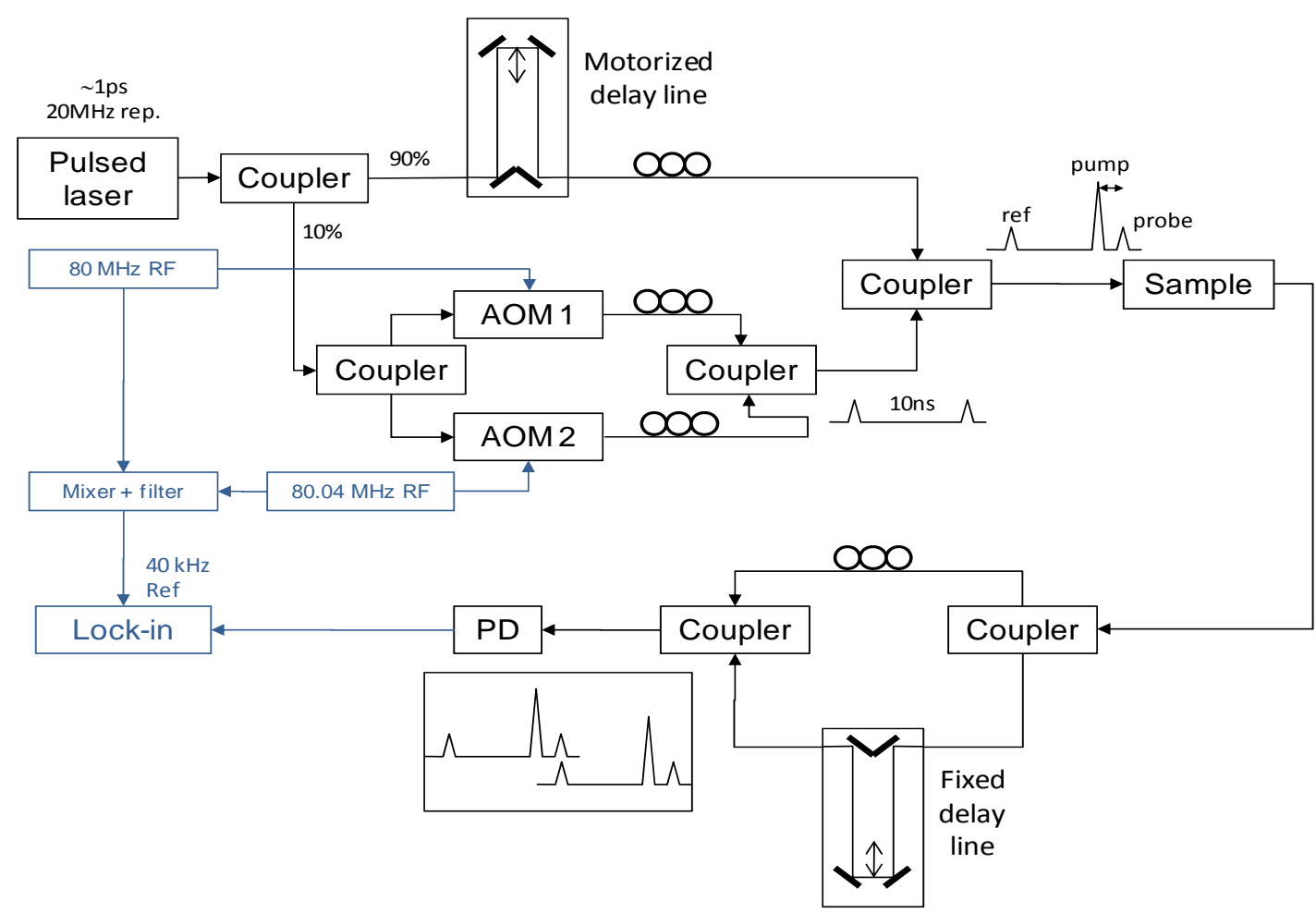

Fig. 5: Pump-probe phase sensitive set-up. Before the sample the input train consists of a probe, a pump and a reference pulse. After transmitting through the sample, the reference and probe pulses are beaten in time and the beating reference signal is detected by a lock-in amplifier.

effects are expected. Fig. 6 shows the nonlinear responses of the SWNT film-wrapped TFBG, a regular TFBG without coating, and standard fiber. These results demonstrate that interaction of the pump with the nonlinear cladding, as mediated by the TFBG, is sufficient to modulate the amplitude of the probe by more than $6 \%$ (12\% in intensity) on a picosecond time scale. We also have checked that the magnitude of the effect depends on the relative spectra of the TFBG and of the probe light, indicating possible optimization strategies.

\section{Conclusions}

In conclusion, SWNT-coated single mode optical fibers have been proposed using, for the first time, a standard optical fiber with a TFBG for nonlinear applications. Two simple techniques for nanotube deposition on the cladding surface have been carried out. The use of tilted fiber Bragg grating assisted the interaction of transmitted light with the SWNT-coating.

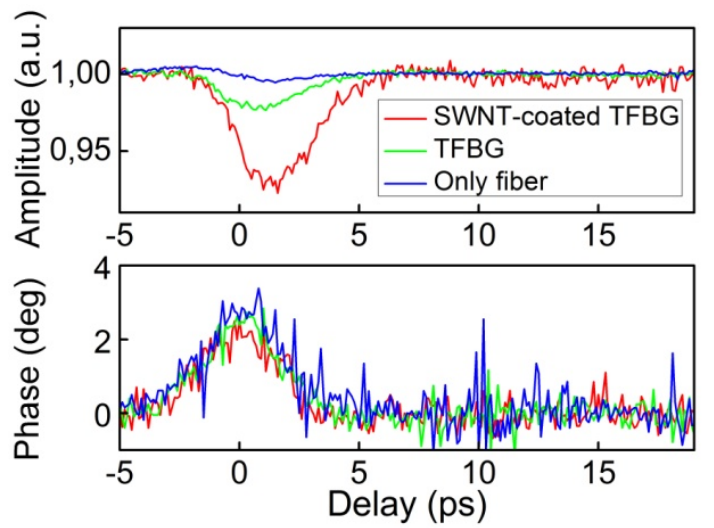

Fig. 6: Nonlinear amplitude and phase responses of the SWNT-coated TFBG, of an un-coated regular 4 deg TFBG and of a standard fiber.

Measurement of cladding mode resonances allowed us to monitor the progressive deposition of nanotubes on the cladding for a dip-coating method. The wrapping method provided a simple and innovative way to deposit thick SWNT layers on optical fibers. Picosecond nonlinear effects were measured by means of pump-probe measurements, showing an 
effective intensity modulation via TFBG cladding mode coupling.

\section{Acknowledgements}

This work was financially supported by the European Commission under the FP7 EURO-FOS NoE (ICT-2007-2-224402) and the Natural Sciences and Engineering Research Council of Canada (NSERC). The work of G. E. Villanueva was supported by the MEC-FPU programs. The work of P. Pérez-Millán was supported by the Juan de la Cierva program, JCI-2009-05805. 